\title{
Multivaluedness in Cellular Automata with Strong Anticipation and Some Prospects for Computation Theory
}

\author{
ALEXANDER MAKARENKO \\ Institute of Applied System Analysis \\ National Technical University of Ukraine \\ (I. Sykorski Kiev Politechnic Institute) \\ 37 Prospect Peremogy, building 35, 03056, Kyiv-56 \\ UKRAINE \\ makalex51@gmail.com
}

\begin{abstract}
It is considered the general formulations and properties of cellular automata with cells which have the strong anticipatory property (introduced by D. Dubois). Multivalued behavior (hyperincursion) of solutions of such CA is described. It was posed new research problems of computation theory related to presumable multivaluedness of cellular automata with strong anticipation property. Extending of classical automata, Turing machine and algorithms had been proposed. Also some relation of such cellular automata and quantum mechanics are discussed.

Key-Words: cellular automat, nature-inspired, strong anticipation, multivalued solutions, hypercomputation, automata, algorithms,
\end{abstract}

Received: October 20, 2019. Revised: April 16, 2020. Accepted: April 22, 2020. Published: April 28, 2020.

\section{Introduction}

The history of cellular automata (CA) concept has more than 50 years development origin formally from the works of S. Ulam and J. von Neumann (see the reviews at [1 - 7]). During the development of CA concept the formalization of so called classical CA had been proposed. Conditionally the classical $\mathrm{CA}$ is defined on regular lattice of cells; each cell has finite number of states and transition roles for changing the states exist. The next in time state of cell depends on cell's states from sum neighborhood of cell. Recently the number of investigations as theoretical as applied had been proposed with classical CA (see for example papers in J. of Cellular Automata, Proceeding of ACRI, CiE, AUTOMATICA conferences, subsections in IT, physical and mathematical conferences etc.).

At the same time many development of CA concept have been proposed. Here we remember only some of them: quantum automata; $\mathrm{CA}$ with special properties (for example non-deterministic); CA with extended spaces for cell's states (fuzzy, complex numbers, p-adic, 'grossones'), CA on non-regular spaces, non-local CA, cellular automata on networks [8 - 11]. Also we should remember on CA with the memory [12] and hierarchical CA [13]. Usually new modifications of CA follow from the new applications of cellular automata. It is evident that further development of CA also will be related to the new fields of investigations.

In given paper we propose the description of new class of CA, namely on the cellular automata with strong anticipation property. Roughly speaking the anticipatory property consists in dependence of transitions to next states on presumable future states of the system. This property is some counterpart to memory property. One of the most prospective ideas is strong anticipation introduced be Daniel Dubois from Liege (Belgium) [14, 15]. Remark that strong anticipation is necessary in large socio-economical systems investigation [16]. Remark that the anticipation in cellular automata had been introduced in papers by D. Dubois and further for example had been considered by Y. Gunji with colleagues [17]. Further investigations allow to consider the new possibilities in multivaluedness origin in cellular automata, for example in modifications of classical "Game of Life" $[18,19]$. In follows to emerging new possibilities for behaviour of such cellular automata with strong anticipation. Of course proposed results of investigations are only the first steps in considering the properties of such CA and in the presumable interpretations of the solutions. So in proposed paper we give only initial description of such CA, few illustrative examples of the solutions and many propositions for further research problems.

Presumable multivaluedness of the solutions of CA with strong anticipation is one of the main new phenomena in such CA. It is well known that classical CA has important influence on many fields of science and technology. Especially interesting are contributions to the computation theory. The first fact is that classical CA can emulate each Turing machine. Also almost all of the objects of computational theory have discrete nature. So, 
classical CA as discrete models may be used in computational theory problems. CA concept is important for evolution modeling in biology and artificial life.

Presumable multivaluedness of CA opens new possibilities for reconsidering existing development of computation theory. The multivaluedness may increases the speed of computation and the capacity of memory. Also multivaluedness of new CA allows introducing the new interpretation of solutions of such models. Of course many future investigations will be needed to understand the new possibilities. But in present paper we introduce only the first considerations of new research problems related to presumable multivaluedness of CA. The structure of the paper is the next. Because of not well-known results on strong anticipation and CA with strong anticipation for completeness we first of all give the description in sections 2 and 3. At first we describe the system with strong anticipation. Then in section 4 we describe some examples of $\mathrm{CA}$ with anticipatory property and their solutions multivalued behavior. Finally in section 5 we propose some further prospects for investigations related to the CA with strong anticipation. Two presumable relation may be especially interesting - to the computation theory and to the quantum mechanical description.

\section{Cellular automata with anticipation}

\subsection{Classical cellular automata description}

Here we give the formal description of cellular automata with anticipation. First of all we follow the papers and books on CA (see for example [1-7]). We pose here the description of one-dimensional CA from [7, p.2] but the description can be modified to many-dimensional case.

'One-dimensional CA is represented by an array of cells $x_{i}$ where $i \in \Sigma$ (integer set) and each $x$ takes a value from a finite alphabet $\Sigma$. Thus, a sequence of cells $\left\{x_{i}\right\}$ of finite length $n$ represents a string or global configuration $c$ on $\Sigma$. This way, the set of finite configurations will be represented as $\Sigma^{n}$. An evolution is represented by a sequence of configurations $\left\{c^{t}\right\}$ given by the mapping $\Phi: \Sigma^{n} \rightarrow \Sigma^{n}$; thus their global relation is following

$\Phi\left(c^{t}\right) \rightarrow c^{t+1}$

where $t$ is time step and every global state of are $c$ is defined by a sequence of cell states. Also the cell states in configuration $c^{t}$ are updated at the next configuration $c^{t+1}$ simultaneously by a local function $\varphi$ as follows'

$\varphi\left(x_{i-r}^{t}, \ldots, x_{i}^{t}, \ldots, x_{i+r}^{t}\right) \rightarrow x_{i}^{t+1}$

Also for further comparing and discussion we show the description of CA with memory from [7, p.3]:

'CA with memory extends standard framework of CA by allowing every cell $x_{i}$ to remember some period of its previous evolution. Thus to implement a memory we design a memory function $\phi$, as follows:

$\phi\left(x_{i}^{t-\tau}, \ldots, x_{i}^{t-1}, x_{i}^{t}\right) \rightarrow s_{i}$

Such that $\tau<t$ determines the degree of memory backwards and each cell $s_{i} \in \Sigma$ is a state function of the series of the states of the cell $x_{i}$ with memory up to time-step. To execute the evolution we apply the original rule as follows:

$\varphi\left(\ldots, s_{i-1}^{t}, s_{i}^{t}, s_{i+1}^{t}, \ldots\right) \rightarrow x_{i}^{t+1}$

In CA with memory, while the mapping $\varphi$ remains unaltered, historic memory of all past iterations is retained by featuring each cell as a summary of its past states from $\phi$.'

\subsection{Strong anticipation property}

The term 'anticipation' had been introduced in active using in biology and applied mathematics by Robert Rosen [17]. So this name is well known for common researchers. Less known is contribution by Daniel Dubois to strong anticipation. Since the beginning of 90-th in the works by D. Dubois - see $[14,15,18]$ the idea of strong anticipation had been introduced: "Definition of an incursive discrete strong anticipatory system ...: an incursive discrete system is a system which computes its current state at time $t$, as a function of its states at past times $, \ldots, t-3, t-2, t-1$, present time, $t$, and even its states at future times $t+1, t+2, t+3, \ldots$

$x(t+1)=A(\ldots, x(t-2), x(t-1)$

$, x(t), x(t+1), x(t+2), \ldots, p)$

where the variable $\mathrm{x}$ at future times

$t+1, t+2, t+3, \ldots$ is computed in using the

equation itself.

Definition of an incursive discrete weak anticipatory system: an incursive discrete system is a system 
which computes its current state at time $t$, as a function of its states at past times $, \ldots, t-3, t-2, t-1$, present time $t$, and even its predicted states at future times $t+1, t+2, t+3, \ldots$

$$
\begin{aligned}
& x(t+1)=A(\ldots, x(t-2), x(t-1), \\
& \left.x(t), x^{*}(t+1), x^{*}(t+2), \ldots, p\right)
\end{aligned}
$$

where the variable $x^{*}$ at future times $t+1, t+2, t+3, \ldots$ are computed in using the predictive model of the system" [14, p. 447]. Thus as the further research problem in the field of CA it should be considered system with strong anticipation.

The equations (5), (6) are rather new mathematical objects and their full considerations are the tasks for further investigations. Remark those systems with anticipation (especially strong anticipation) early have been investigated in many research fields: biology, computer science, social sciences, physics, neurophysiology etc. (see Journal of Computing Anticipatory Systems, Proceedings of Conferences CASYS). Because of importance of such systems below we describe one of the tools for their investigations - CA with strong anticipation.

\subsection{Cellular Automata with Strong Anticipation}

The key idea is to introduce strong anticipation into CA construction. Of course many ways exist for implementation such idea. First of all we will describe one of the simplest. Then we will discuss the results of some computer experiments with such CA. For such goal we will suppose that states of the cells of CA can depend on future (virtual) states of cells. Then the modified rules for $\mathrm{CA}$ in one of possible modifications have the form:

$$
\begin{aligned}
& \phi\left(\bar{x}_{i}^{t-\tau}, \ldots, \bar{x}_{i}^{t-1}, \bar{x}_{i}^{t},\right. \\
& \left.\bar{x}_{i}^{t+1}, \ldots, \bar{x}_{i}^{t+k}\right) \rightarrow \bar{s}_{i}^{t+k} \\
& \bar{\varphi}\left(\ldots, \bar{s}_{i-1}^{t+k}, \bar{s}_{i}^{t+k}, \bar{s}_{i+1}^{t+k}, \ldots\right) \\
& \rightarrow\left(\bar{x}_{i}^{t+1}, \bar{x}_{i}^{t+2}, \ldots, \bar{x}_{i}^{t+k}\right)
\end{aligned}
$$

where $k$ (integer) is horizon of anticipation. But because of presumable properties of such solutions (multi-valuedness, see examples and discussion below) the values of variables in (7), (8)) have different interpretations. This is connected with the fact that equation (7), (8) are nonlinear equations on the values of cell's states in future moments of time. Such equations can have one solution, no solutions and many solutions in dependence on parameters and the current and past states of the cells). So in equations (7), (8) $\bar{x}_{i}^{t+j}$ represent all presumable values of cell $i$ at moment $t+j$ and $\bar{s}_{i}^{t+k}$ represent all pre-history (multi-valued) for cell $i$ till the moment $t+k$. Remark that in some particular cases (one - valued solutions of (7), (8)) the results correspond to such in equations (3), (4).

Further we for simplicity describe the system of such CA without memory and only with one-step anticipation $(k=1)$. The general forms of such equations in this case are:

$$
\begin{aligned}
& \phi\left(\bar{x}_{i}^{t}, \bar{x}_{i}^{t+1}\right) \rightarrow \bar{s}_{i}^{t+1} \\
& \varphi\left(\ldots, \bar{s}_{i-1}^{t+1}, \bar{s}_{i}^{t+1},\right. \\
& \left.\bar{s}_{i+1}^{t+1}, \ldots\right) \rightarrow \bar{x}_{i}^{t+1}
\end{aligned}
$$

The main peculiarity of solutions of (9), (10) is presumable multi-validness of solutions and existing of many branches of solutions. This implies also the existence of many configurations in CA at the same moment of time. Remark that this follows to existing of new possibilities in solutions and interpretations of already existing and new originating research problems.

\section{Game 'Life' with strong anticipation.}

In this subsection we propous very short description of computer experiments with one of simple CA with strong anticipation - game 'Life_A'. Such descriptions and illustrations are need for understanding the discussion in the section 5. More details are described in our publications $[19,20]$.

\subsection{Example of cellular automata}

Let's consider rules driving the simple classical "Life" $[1-4,19,20]$ with two possible states of the cells marked as ' 1 ' and ' 0 '. To formalize the transitions roles, we have introduced two auxiliary functions $\mathrm{f}_{0}$ and $\mathrm{f}_{1}$ for transition from such states. Their values may be represented by different ways. Here we give below representation by the table form.

Table 1. Auxiliary functions $\mathrm{f}_{0}(\mathrm{x})$ and $\mathrm{f}_{1}(\mathrm{x})$.

\begin{tabular}{|l|l|l|l|l|l|l|l|l|l|}
\hline $\mathrm{X}$ & 0 & 1 & 2 & 3 & 4 & 5 & 6 & 7 & 8 \\
\hline $\mathrm{f}_{0}(\mathrm{x})$ & 0 & 0 & 0 & 1 & 0 & 0 & 0 & 0 & 0 \\
\hline $\mathrm{f}_{1}(\mathrm{x})$ & 0 & 0 & 1 & 1 & 0 & 0 & 0 & 0 & 0 \\
\hline
\end{tabular}


Suppose that out of the interval $[0 ; 8]$ both these functions are zero-valued. Moreover, if the argument is not an integer value, it is rounded to the nearest integer.

Under the introduced notions next state function for $k$-th cell will be as follows:

$F_{k}=F\left(S_{k}\right)=\left\{\begin{array}{l}f_{0}\left(S_{k}\right), C_{k}=0 \\ f_{1}\left(S_{k}\right), C_{k}=1\end{array}\right.$,

$F_{k} \in\{0,1\}$

where $\mathrm{S}_{\mathrm{k}}$ - number of (non-zero) neighbors of $k$-th cell, $C_{k} \in\{0,1\}$ (usually 0 interprets as if $k$-th cell is dead, 1 - otherwise).

Thus, the evolution of an automaton with $\mathrm{N}$ cells may be denoted as:

$C_{k}^{t+1}=F_{k}^{t}=F\left(S_{k}^{t}\right), k=1 . . N$,

where $\mathrm{t}$-discrete time.

\subsection{Cells with anticipation}

To endow a model with anticipation property one has to design a next step function that depends on the next (expected, virtual) cell states [19, 20]. Many possibilities exist to implement it. We took in $[19,20]$ as the illustrative example for CA with anticipation the case when the next state function of classical "Life" defined as:

$F_{k}^{t}=F\left(S_{k}^{t}\right)$

had been replaced by:

$F_{k}^{t}=F\left((1-\alpha) \cdot S_{k}^{t}+\right.$

$\left.\alpha \cdot S_{k}^{t+1}\right), \alpha \in[0 ; 1]$

or

$F_{k}^{t}=F\left(S_{k}^{t}+\alpha \cdot S_{k}^{t+1}\right), \alpha \in I R$

Such rules correspond to 'integral' accounting the future virtual states by counting only number of presumably occupied cells in the neighbourhood of the cell at future moment of time. Further, these two variants of a next state function will be referred to as weighted and additive, correspondingly. Cellular automata driven by either of these functions were implemented as the computer program.

\subsection{Examples of computer experiments}

Below we pose for illustration of presumable behaviour of such game 'Life_A' with anticipation only one picture. The figure below corresponds to the case with 16 cells with periodic boundary conditions.

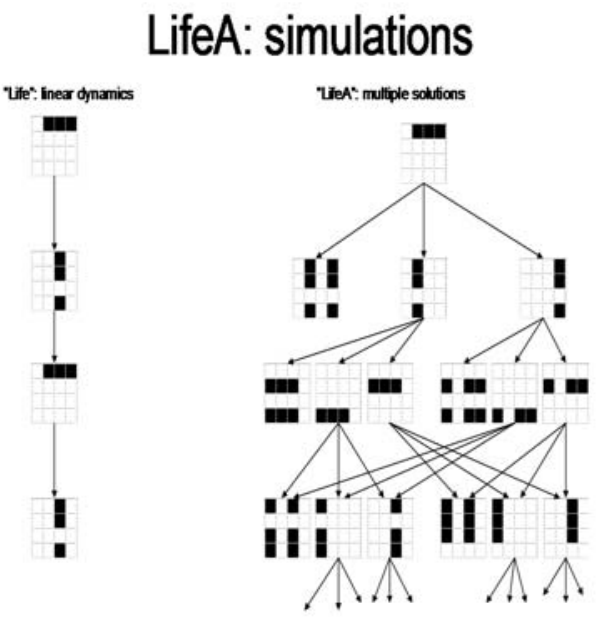

Fig. 1 Comparison the solutions of classical game 'Life' (left side) and Of game 'Life_A' with strong anticipation (right side).

Only 3 discrete time steps are represented at Fig. 1 and at right side of the figure we see possible branching of the solution, existing of many values of cellular's state and multiplicity of configurations (more examples see at $[19,20]$ ). So we can anticipate that such kind of behaviour is typical to CA with strong anticipation. The figure below shows other example of such solution:

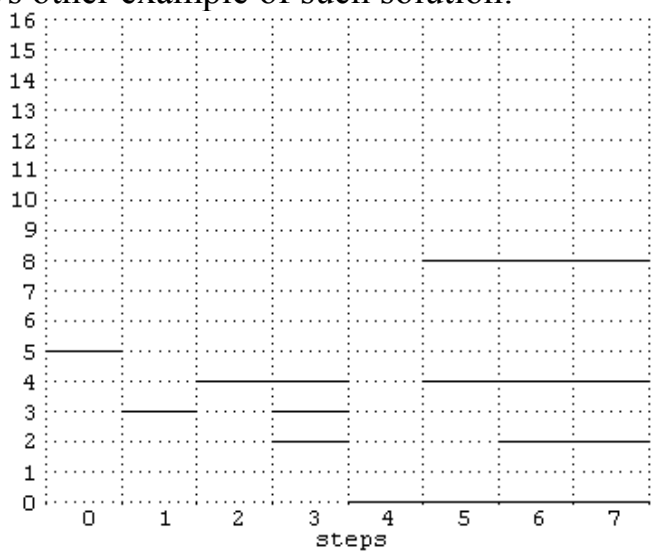

Fig. 2. Solution with multiple states (on abscissa discrete time steps, and on the vertical axis the number of different system configurations (different sets of states of all cells)).

Remember that configuration at given time step are collection of all states of cells. In our example the single configuration corresponds the states of 16 cells. In Fig. 2 the number of realized at given time moment possible states is equal to 4 . Thus, the main distinguishing feature of cellular automata with strong anticipation is the multiplicity of solutions, and multivaluedness of cell's states and of configurations at some time steps. Possible consequences of such properties and presumable 
reconstruction of the theory of automata are proposed at the following sections.

\section{Discussions and further research problems}

\subsection{Presumable development of the theory of cellular automata}

In order to discuss the possible consequences of the new multivalued solutions we first of all will analyse the evolution of the computation theory and applications. After the initial period of automata theory development the next stage came in connection with the study of the game "Life" by J. Conway. It was found many types of solutions of cellular automata, relation with the general theory of automata, the connection with the theory of grammars and languages, etc. The next stage began with the involvement of cellular automata studies the methods of physics, primarily statistical physics. Starting of the application of physical methods for cellular automata in the $80 \mathrm{~s}$ had been associated with the names S. Wolfram, U. Frish, S. Toffoli, B. Chopard et al for the simulation of hydrodynamics. Attractors have been studied in parallel, for local and global maps, languages and machines related to cellular automata, the possibility of using the CA algorithms and programs, etc. Both deterministic and probabilistic CA and CA were constructed with a complicated structure (non-local, second-order (hierarchical), heterogeneous, with memory).

Next essentially new stage can be roughly related to the development of the theory of quantum computing including quantum cellular automata. For conditional beginning of this stage usually takes the appearance of articles R. Feynman $(1982,1986)$ for quantum computation [21]. Of the fundamentally important accomplishments are the formulation of the concept of a quantum machine, including CA, quantum Turing machines, quantum computation and logic applied to other quantum CA promising formulation local aspects $\mathrm{CA}$ in terms of local algebras of operators in the spirit of the results G. Haag.

Currently, all of the above areas continue to evolve (see works by C. Moor, P. Cratchfield, S. Toffoli, B. Chopard and many others). Along the way are becoming increasingly popular and the use of CA [22]. Moreover, in view of hypotheses about the cellular structure of space-time at the micro-level (the Planck length, D. Finkelshteyn's work (1973) and many others), sometimes it is declared that the universe is a giant cellular automaton with appropriate computational processes - see the book by S. Wolfram 2002 [1].
Note that all the development of cellular automata occurred approximately as follows - there were certain concepts (such as classical or quantum computing) which then is embedded into the concept of cellular automata. Of course, the proposed cellular automata with anticipation [19, 20] partially admit such an immersion into existing classical concepts. We call it the direct way of theory development. However, namely the solutions of the new CA models open the new paths for development of first of all theory that can be called the 'inverse way' or 'reconstruction of concepts'. We discuss some of such possibilities in the next subsections.

\section{Problem of the reconstruction of concepts}

Again, now the development of cellular automata, both classical and quantum follows to "direct" way of development. That is, more often there were original concepts, then the CA had been proposed for concrete problems, and then their properties and applications are studied. Proposed CA leads to the known equivalent representations (languages, automata, dynamical systems). All this is discussed in the framework of a predetermined structure of space- time. We can see that our research on CA with anticipation (and subsequent generalizations) leads to possibilities of reconsidering classical problems.

There is a new source of problems, concepts, generalizations and interpretations. We call this way "reverse" in research or by "reconstruction" concepts. It comes from the fact that the primary, source, the basic element of the CA unit cell is given with its laws of evolution states. We assume that the existence of the enveloping concepts (meta systems) and others with known properties were not originally supposed to, and they can be built, removed, identified only as properties of solutions of CA system. For example, for conventional CA were aware that such a machine, language, dynamic system, equivalence, etc. The introduction of anticipation in CA leads to ambiguity in the decisions (we call these cellular automata with multivaluedness). To specify multiple meanings will add the letter $\mathrm{M}$ to the corresponding object. Then it should be introduced the concepts of the theory of automata with multivaluedness (AM). Turing machine (TM) which allows ambiguity may be marked as (TMM), algorithms with multivaluedness - (AlgM), formal languages with multivaluedness as (LM). Then it is necessary to revise the Turing thesis - Church on computability by means of 
automata (TChM), Turing test for artificial intelligence (AIMtest).

In physics, this leads to the possibility of considering the multi-valued statistical mechanics (SMM); multivalued cosmology (KM), multi-valued quantum mechanics (CMM) and the multiple structures of matter, etc. Note that Everett's interpretation of quantum mechanics can be useful for such considerations. Conversely, multi-valued CA can help in understanding Everett picture of the Universe. It seems that the new opportunities and understanding of the nature of probability can be proposed. More narrowly, it may lead to new research on multiple-valued solutions in CA and evolutionary objects: multivalued chaos, cycles, bifurcations, Markov chains, attractors.

It's all relatively obvious examples of 'reconstruction' concepts. But no limits for generalizations exist. In fact, the structure of the "reverse" direction of research in CA may follows to generalized elements with multiplicity. Therefore, generalizations, first, may concern to the state spaces of CA (or configurations). Also integer and real numbers can changes on other options (nonstandard analysis, p - adic analysis; $\infty$ - structure, logical calculus; topological objects (e.g. homology) distribution). Well, then state operators, secondary operators (operads), etc can be proposed. To all this diversity more general rules - generalized operators, multi-valued, discrete or continuous delay (and ahead) can be considered. There may be introduced different definitions of neighbourhoods in CA (including implicit, fuzzy, time-dependent and state). Further generalizations can be viewed by using the CA as CA cells with elements of artificial intelligence. Thus, elements of the CA can be self reflective elements with infinite recursion level. Each proposed architecture can induce construction of new structures, etc. We note only that the obvious way on this path suggests language of categories and functors (including facilities for the study of the limit), which is becoming a commonplace in theoretical computer science and quantum field theory. We also point out that many of the issues discussed can also be applied to the model equations in the form of another type (e.g., differential).

Some features of cellular automata with strong anticipation are described in [23]. We stress here only some interesting features. First, we mention the application to the theory of automata and cellular automata. Cellular automata with strong anticipation can implement non-classical logic and may be nonTuring machines, which is important for hypercomputation. In particular, it can be implemented possible worlds approach S. Kripke and J. Hintikka, as well as in line with the ideas N. Belnap. Interesting is the consideration of the implementation of computers by strong anticipative elements. Also it is possible to consider the issues in the consciousness and effects on cognitive science [24].

\subsection{Some problems in the theory of computations in terms of the effect of strong anticipation}

In given subsection we reconsider some concepts from computational theory from the point of view of strong anticipation theory.

\subsubsection{Automata theory.}

Let us recall the basic concepts of classical automata theory Definition 1.37 [22]:

The non-deterministic finite state machine is 5-tuple $\left(Q, \Sigma, \delta, q_{0}, F\right)$, where

1. $Q$ finite set of states

2. $\Sigma$ finite alphabet

3. $\delta: Q \times \Sigma_{\varepsilon} \rightarrow P(Q)$ is a transformation function

4. $q_{0} \in Q$ initial state, and

5. $F \subseteq Q$ There are many admissible states

Automata as a whole are divided into deterministic and non-deterministic [21, 22]. For our purposes, it is especially important to define nondeterministic automatic machines. The main difference is that the transition function $\delta$ in case of CA with strong anticipation is multivalued, where $P(Q)$ some (not necessarily a one-point) set. Usually it was believed that this is purely empirical (non-physical) construction. Two interpretations of nondeterminism were discussed. According to one version, the tree of transitions is considered, and according to the second, in the case of ambiguity the automaton splits into a number of its copies. However, both were considered as purely hypothetical constructions.

Let's consider what a strong anticipation can give in terms of interpretation of the non-deterministic version of the machine. Assuming that systems with strong anticipation are actually realized in nature (discussion of various possibilities in some papers by author) we consider below some presumable consequences.

First, if the number of branches of an automaton with strong anticipation is finite, then such an automaton may be just the realization of a finite nondeterministic automaton. But the structure of the solution set of a system with strong anticipation 
provides much more possibilities than classical automata. Such elements admit an infinite number of simultaneous states. This, at least follows to infinite automata. Recall that the potential advantage of nondeterministic finite automata is their greater efficiency than deterministic, although it is proved that for each nondeterministic automaton it is possible to construct an equivalent deterministic (M. Rabin, D. Scott). However, it seems that such a proof does not apply for case of infinite automata. At the same time, in the case of infinite automata with anticipation, the potential computational capabilities of nondeterministic automata are preserved in any case, which in particular can be useful for solving NP completeness problems.

\subsubsection{The universal Turing machine.}

The next aspect of questions in the theory of computation is related to the concept of a Turing machine. Briefly, the classical Turing machine is described as follows [21, 22]:

Definition 3.3 The Turing machine has a 7-tuple, $\left(Q, \Sigma, G, \delta, q_{0}, q_{\text {accept }}, q_{\text {reject }}\right)$, where all finite sets and

1. $Q$ There are many states,

2. $\Sigma$ There is an input algorithm that does not contain an empty character,

3. $G$ is a tape alphabet, where $W \in G$ и $\Sigma \subseteq G$, 4. $\delta: Q \times G \rightarrow Q \times G \times\{L, R\}$ there is a transition function

5. $q_{0} \in Q$ is an initial state

6. $q_{\text {accept }} \in Q$ is a state of acceptance

7. $q_{\text {reject }} \in Q$ is a state of denial, where

$q_{\text {accept }} \neq q_{\text {reject }}$

It is important that for classical cellular automata their computational equivalence of the classical Turing model is established [21, 22]. In this case, computational architecture had been proposed, using both the basic architecture of cellular automata for register emulation and general properties.

What can a strong anticipation do in terms of the theoretical construction of the computing process? It is possible to construct analogues of classical designs with allowance for strong anticipation. To do this, you must enter analogues of computer elements - registers, stacks, communications, etc. for a multi-valued case, but this requires separate complex constructions. However, there is a relatively simpler possibility - to use the idea of attracting "gliders" [1-5] to emulate logical operations and the computational process. It is very important that many-valued analogues of gliders are also found in cellular automata with strong anticipation (for the one-dimensional case see [24]), and two-dimensional analogues in D. Krushinsky's thesis).

It is well known that in case of classical cellular automata any Turing computer can be emulated by gliders in cellular automata [1] (see the case C in Figure 3). Then many-valued gliders can replace classical single-valued gliders. In this case, it becomes possible to illustrate the mass of new cases that were not even supposed to happen in the classical case (see Figure 3):

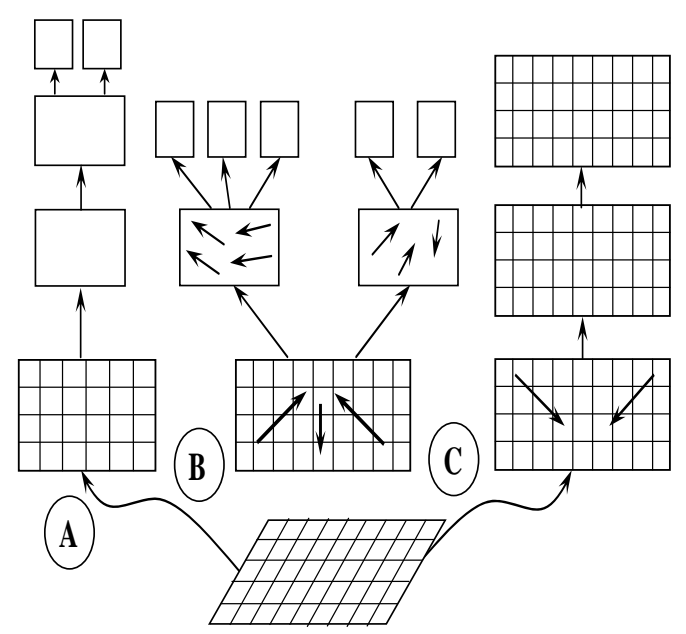

Fig. 3. Hypothetical solutions with gliders in different branches.

Gliders are conventionally marked with arrows in rectangles. The branch A corresponds to the transition from a single-valued branch to two-valued case at some time, the case of branch B corresponds to a continuous increase in the number of branches, and the case of branch $\mathrm{C}$ to the usual single-valued case.

The figure gives an illustration of the hypothetical case, when at first from some time $t$ to $t_{1}$ there is a unique evolution with classical gliders. Then, at the moment $t_{1}$, a multi-valued case (branching) of configurations, possible at the same time (we designate the branches as A, B, C) occurs. In this variant (Figure 3 ) in each of the fields shown in the Figure in the form of rectangles, the emulation of its Turing machine is calculated. However, more complex cases are possible - the existence of an infinite number of emulations (branches) simultaneously, the merging of emulations, the collision of emulations, specially selected algorithms for mergers, multiplications, etc. True, 
here a question arises that is common to all subjects with multiple meanings: languages that are recognized; that they can calculate such computers; what can be measured on the basis of multi-valued systems; optimization of computation processes, the task of synthesizing the structures of a computational element and computing systems, and the like. Also existing of multivaluedness and of different branches allows to try to reconsider the problem of parallel computation.

\subsubsection{The theory of computations and algorithms.}

An important part of the classical theory of computations is the theory of algorithms and recursive functions. At present, there are quite a lot of definitions of algorithms, among which the most well-known (or, say, accepted): A. Markov, A. Kolmogorov, G. Chaitin, Yi. Moschovakis, Y. Matiyasevich, Y. Gurevich [21, 22]. Usually a formal description of algorithms requires a lot of space. Therefore, here we note one main feature the recognized inseparable connection of classical algorithms with their feasibility by classical Turing machines. For example (from Wikipedia: http://en.wikipedia.org/wiki/Algorithms). "Thus, an algorithm can be considered to be any sequence of operations that can be simulated by a Turingcomplete system. Authors who assert this thesis include M. Minsky (1967), J. Savage (1987) and Y. Gurevich (2000):

Minsky: "But we will also maintain, with Turing ... that any procedure that could" naturally "be called effective, can in fact be realized by a (simple) machine. its favor are hard to refute. "

Gurevich: "... Turing's an informal argument in favor of his thesis that justifies a stronger thesis: every algorithm can be simulated by a Turing machine ... according to J. Savage [1987], an algorithm is a computational process defined by a Turing machine" . ")

The above considerations also make it possible to raise questions about the expansion of the notion of algorithms by introducing a larger fraction of polysemy into basic definitions, admitting the multivaluedness of the elements of the relationship, and so on.

Of course, the issues indicated in this section require further in-depth consideration, especially regarding the possible equivalence / non-equivalence with classical cases. However, due to the potential ambiguity, in any case, we can expect an acceleration of the calculations.

\subsubsection{Some correlations with quantum- mechanical aspects}

Geometry, beginning with the development of modern science, and especially since the time of Newton enters theoretical physics in one way or another. One of the key questions is the choice of spaces, in terms of which they formulate the corresponding equations and sets of values that can take physical quantities and variables.

Up to now, usually the basic equations of quantum mechanics are partial differential equations. It is important to emphasize that with this description it is assumed that the independent variables vary continuously (spatial, temporal). Further, unknown functions, as a rule, were continuous or smooth. This made it possible to use the whole arsenal of mathematical analysis, the theory of differential equations, including their symmetry analysis. As the standard reference see the book of W. Thirring.

However, especially recently, changes in the methods of describing the corresponding mathematical objects began to appear. Examples include p-adic physics (I. Volovich, A. Khrennikov), non-standard analysis (A. Robinson), superphysics (E. Witten), and application of category theory, physical structures (Yu. Kulakov, V. Vladimirov) and others. However, among all the various generalizations, it is necessary to note a direction that combines approaches that take into account both continual and discrete aspects. As is well known [25, 26], the discreteness in quantum mechanics has various aspects, beginning with the fact that there exists a minimal (Planck) length ( $10^{-35} \mathrm{~m}$ ). Using the presence of this scale of length, models are constructed on a lattice with certain limiting transitions with a decrease in the lattice step).

The theory of cellular automata (J. von Neuman, T. Toffoli, S. Wolfram, A. Adamatsky) and classical quantum mechanics (L. Landau, M. Jemmer) are some very well-developed branches of science, which, at first glance, have very little in common. But in the last 20-30 years there were works in which the researchers tried to distinguish points of common (similar) statements and interpretations of the results. But in most approaches, the main thing was to involve the approach of cellular automata to the problems of quantum mechanics (see G. Grossing, A. Zellinger, A. Aspect, A. Bisio, D'Ariano, G. t'Hooft). This was due to the fact that, in cellular automata, and in quantum mechanics, it is fundamentally important is the use of the discreteness of space and behavior in one or another of its manifestations. Some very interesting application of CA with quantum properties are in 
[27]. The author also described some aspects of the application of CA to quantum systems [28]. The special emphasis had been placed on the possible role of strict anticipation for such problems.

Recently a reverse trend has also emerged - that is, an understanding of what quantum mechanics can provide for tasks in the field of cellular automata. Note that there are still very few works of this kind. One of the first in this area is the study of quantum cellular automata, which may be important for the investigation of quantum cellular automata for quantum computers [23].

Consideration of the possibilities of $\mathrm{CA}$ with a strong anticipaition already revealed interesting new opportunities $[19,20]$. But in parallel there are papers on the transfer of the problems of classical quantum mechanics to the CA field. So, interesting work was done on the interpretation of a two-slit experiment on the diffraction of quantum particles on a screen with two gaps on the behavior of solutions of media models consisting of classical cellular automata (or even living cells!) (A. Adamatski).

Proceeding from our previous research on cellular automaton models with the property of strong anticipation $[19,20]$, it can be assumed that the modification of the two-slit problem in case of strong anticipation will clarify important issues.

\section{Conclusions}

Thus at this paper we described the new cellular automata with strong anticipation behavior. The main new aspect is presumable multivaluedness of the solutions. Presumable multivaluedness opens absolutely new possibilities for receiving new interesting results as in CA behaviour as in interpretations of presumable solutions. Here we describe only few of such presumable possibilities, some of which already are under investigations.

1. At first we remember the new possibilities in considering of non-deterministic CA (and moreover usual automata). Non-deterministic automata allow few transition ways from one state to others. Usually it is supposed that such structure is only theoretical and in reality only one of the ways is used in each transition. CA with strong anticipation opens the natural possibility for considering of the systems with many different ways in parallel. Accepting possibilities of physical realization of strong anticipatory systems it may be accepted existence of CA with many branches. Also such systems are interesting as multi-valued dynamical systems.

2. In proposed paper we have considered only the case of finite alphabet for indexing the cell's states.
But previous investigations of dynamical systems with strong anticipation show the possibilities of existing of the solutions with infinite numbers of solution branches. This allows introducing CA with infinite number of cell' states (or at least infinite alphabet for CA).

3. The generalizations from point 1 and 2 at this subsection and analysis of automata and CA theories origin follows to presumable considering of some aspects of computation theory. The short list of topics may be the next: computability; Turing machines; automata and languages; recursive functions theory; models of computation; new possibilities for computations with accounting possible branching.

4. One of the important results is the possibility of the emergence of multi-valued solutions. An immediate consequence is the possibility of posing problems of multivalued computation machines, languages, chaos, etc.

5. Also we considered the use of models of cellular automata in quantum mechanics and the possible ways of developing such representations. As one of the possible generalizations, we consider the introduction of strong antiparticle in some equations for quantum mechanics and possible consequences. One of the most promising opportunities is the emergence of many ways in the solution.

ACNOWLEDGEMENT. The author would like to give the appreciation for D. Krusinsky for some computer realizations of model Life with anticipation.

References

[1] Wolfram S. New kind of science. Wolfram Media Inc., USA, (2002).

[2] Illiachinski A., Cellular Automata. A Discrete Universe. World Scientific Publishing, Singapoure, (2001).

[3] Kari J. Theory of cellular automata: A survey. Theoretical Computer Science, 334, 333 (2005)

[4] Kutrib M. Non-deterministic cellular automata and languages. Int. Journal of General Systems. 41, 555 - 568. (2012)

[5] Adamatskii A., Komosinski M. Artificial Life Models in Software. Berlin, SpringerVerlag. 2005.

[6] Chopard B., Droz M. Cellular Automata Modeling of Physical Systems. Cembridge Univ. Press. 199

[7] Sergeyev Ya.D., Garro A. Single-tape and multi-tape Turing machines through the lens of the Grossone methodology, Journal of Supercomputing, 65(2), 645-663. (2013) 
[8] D'Alotto . Cellular Automata Using Infinite Computations, Applied Mathematics and Computation, 218(16), 8077-8082. (2012).Sipser M. Introduction to the theory of computation. 2013.

[9] Cooper B. Computability theory. Chapman\&Hall, 2004.

[10] Mynski M. Computations and Automata 1967

[11] Facchett L., Makarenko A. 'Game of Life' with Modifications: Non-regular Space, Different Rules and Many Hyerarchical Levels. Int. J. Information Content \& Processing. 2017. Vol. 4, N. 1. Pp. 21-50.

[12] Martinez G.J., Adamatzky A., Alonso-Sanz R. Complex dynamics of elementary cellular automata emerging from chaotic rules. Int. Journal of bifurcation and chaos, 22, 1250023, (2012

[13] Bandini S., Mauri G. Multilayered cellular automata. Theoretical Computer Science, 217, 99-113, (1999).

[14] Dubois D., Generation of fractals from incursive automata, digital diffusion and wave equation systems. BioSystems, 43, 97-114, (1997).

[15] Dubois D., Incursive and hyperincursive systems, fractal machine and anticipatory science. Published by the American Institute of Physics, AIP Conference Proceedings 573, 437 - 451, (2001).

[16] Makarenko A., Anticipating in modeling of large social systems - neuronets with internal structure and multivaluedness. International .Journal of .Computing Anticipatory Systems, 13, 77 - 92. (2002).

[17] Rosen R., Anticipatory Systems. Pergamon Press. (1985).

[18] Dubois D., Introduction to Computing Anticipatory Systems. International Journal of Computing Anticipatory Systems, 2, 3-14. (1998).

[19] Cellular Automata. ACRI-2008. Umeo H., Morishita S., Nishinary K., Komatsuzaki T. LNCS 5191, (2008). pp. 77-82.

[20] Krushinskiy D., Makarenko A., Cellular Automata with Anticipation. Examples and Presumable Applications. AIP Conf. Proc., vol.1303, ed. D.M.Dubois, USA, (2010). pp. 246-254.

[21] Makarenko A. Multivaluedness Aspects in Self-Organization, Complexity and Computatios Investigations by Strong Anticipation. Chapter in Book: Recent Advances in Nonlinear Dynamics and Snchronization. Eds. Kyamakya
K., Mathis W., Stoop R., Chedjou J., Li Z. , Springer; Cham, 2018. Pp.33-54.

[22] Sipser M. Inrtoduction to the theory of computation. Second edition. Thomson Course Technology, USA (2006).

[23] Quantum computer and quantum computing . Vol..2 . Izhevsk, Republican printings, 1999. $288 . \mathrm{p}$

[24] Terpil E., Makarenko A. Simulation of Public Opinion with Ideas of Cellular Automata. In: Wąs J., Sirakoulis G.C., Bandini S. (eds) Cellular Automata. ACRI 2014. Lecture Notes in Computer Science, vol 8751. Pp. 518-528. 2014. Springer, Cham

[25] Landau L., Liphshitc L Quantum Mechanics. 1974. 762 c.

[26] Interpretations of Quantum Mechanics. http://en.wikipedia.org/wiki/Interpretations_of_ quantum_mechanica

[27] Mardies V., Sirakoulis G. Ch., Karafyllidis I. Automated Design Architecture for 1-D Cellular Automata using Quantum Cellular Automata. IEEE Transactions on Computers, vol.64. n.9. pp. 2475-2489, 2015.

[28] Makarenko A. Cellular authomata methods in quantum physics. Collection of papers from the Austro-Ukrainian Seminar at Wienna, January 2016. K.: TIMPANI. 201613 p. (in Russian).

[29] Stepan Bilan, Mykola Bilan, Sergii Bilan, Research of the Influence of the local Transition Function on the Formation of a New Active Cell in the PRNG Based on ACA, WSEAS Transactions on Information Science and Applications, pp.167-173, Volume 14, 2017 